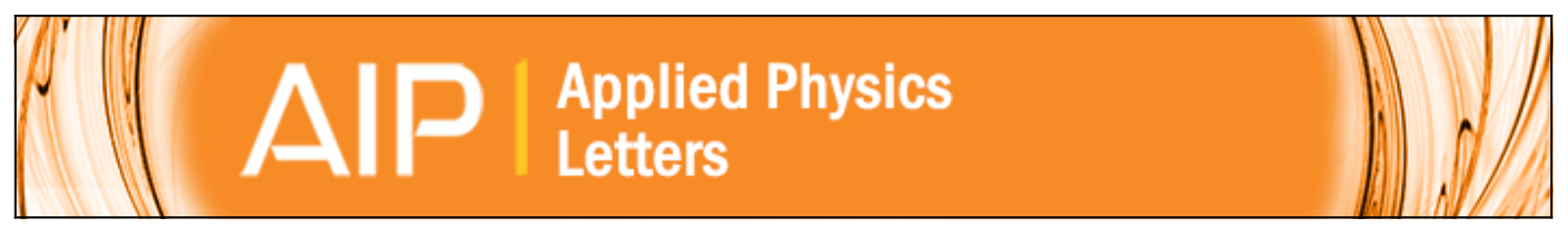

\title{
Bending of electromagnetic waves in all-dielectric particle array waveguides
}

Roman S. Savelev, Dmitry S. Filonov, Polina V. Kapitanova, Alexander E. Krasnok, Andrey E. Miroshnichenko,

Pavel A. Belov, and Yuri S. Kivshar

Citation: Applied Physics Letters 105, 181116 (2014); doi: 10.1063/1.4901264

View online: http://dx.doi.org/10.1063/1.4901264

View Table of Contents: http://scitation.aip.org/content/aip/journal/apl/105/18?ver=pdfcov

Published by the AIP Publishing

\section{Articles you may be interested in}

Dielectric stripes on gold as surface plasmon waveguides: Bends and directional couplers

Appl. Phys. Lett. 91, 081111 (2007); 10.1063/1.2772774

Sharp waveguide bends induced by spatial solitons

Appl. Phys. Lett. 88, 061117 (2006); 10.1063/1.2172649

Bends and splitters in metal-dielectric-metal subwavelength plasmonic waveguides

Appl. Phys. Lett. 87, 131102 (2005); 10.1063/1.2056594

Bending optical solitons in nonlinear photonic crystal waveguides

J. Appl. Phys. 97, 026104 (2005); 10.1063/1.1839650

Highly confined waveguides and waveguide bends in three-dimensional photonic crystal

Appl. Phys. Lett. 75, 3739 (1999); 10.1063/1.125441

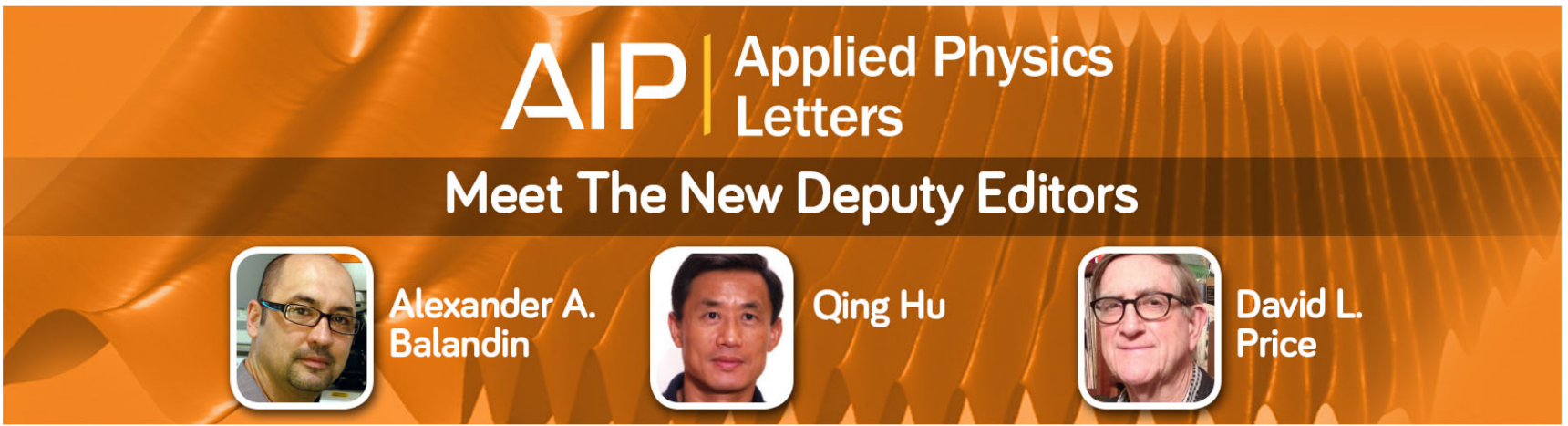




\title{
Bending of electromagnetic waves in all-dielectric particle array waveguides
}

\author{
Roman S. Savelev, ${ }^{1}$ Dmitry S. Filonov, ${ }^{1}$ Polina V. Kapitanova, ${ }^{1}$ Alexander E. Krasnok, ${ }^{1}$ \\ Andrey E. Miroshnichenko, ${ }^{2, a)}$ Pavel A. Belov, ${ }^{1}$ and Yuri S. Kivshar ${ }^{1,2}$ \\ ${ }^{1}$ ITMO University, St. Petersburg 197101, Russia \\ ${ }^{2}$ Nonlinear Physics Centre, Research School of Physics and Engineering, Australian National University, \\ Acton, ACT 2601, Australia
}

(Received 12 August 2014; accepted 28 October 2014; published online 7 November 2014)

\begin{abstract}
We propose and demonstrate experimentally an alternative approach for realizing subwavelength photonic structures, exploiting the waveguiding properties of chains of high-index dielectric disks with both electric and magnetic dipole resonances. We reveal that the electromagnetic energy can be efficiently guided through sharp corners by means of the mode polarization conversion at waveguide bends. We confirm experimentally the guidance through a $90^{\circ}$ bend in the microwave frequency range. (C) 2014 AIP Publishing LLC. [http://dx.doi.org/10.1063/1.4901264]
\end{abstract}

A design of highly efficient integrated photonic circuits with combined optical and electronic components for the subwavelength guiding of the electromagnetic energy is one of the main trends of the modern nanophotonics. ${ }^{1-3}$ In order to achieve high integration densities, optical waveguides with subwavelength light localization have been proposed. Conventional silicon wire waveguides have a minimal crosssection size, and they can be manufactured with high quality. ${ }^{4-6}$ However, such waveguides do not provide low-loss propagation of optical signals through sharp bends, and they require rather large bending geometries thus increasing the overall size of a photonic chip. ${ }^{7}$ Photonic crystals have been viewed as a possible alternative, and it has already been demonstrated that light can be guided by a waveguide composed of defects, and such waveguides can have sharp bends. ${ }^{8-10}$ However, the property of photonic crystals to transmit light through sharp bends was found to depend strongly on the bend geometry being also linked to the strict resonant conditions associated with the Fano resonance where the waveguide bend plays a role of a specific localized defect, ${ }^{10}$ thus demonstrating narrowband limited functionalities.

Another candidate for the efficient propagation through sharp bends is a coupled-resonator optical waveguide (CROW) ${ }^{11}$ The adiabatic coupling, as suggested in Ref. 11, between resonators requires relatively large sizes, which are not suitable for chip integration. Similar waveguides can be realized as chains of metallic nanoparticles guiding the electromagnetic energy via localized suface plasmon resonances. ${ }^{12-14}$ Theoretical studies in the quasistatic single-dipole approximation suggested an efficient power transmission through sharp bends in such waveguides. ${ }^{15,16}$ Small size of nanoparticles (several tens of nanometers) made these waveguides very attractive for nanophotonic applications. However, later studies revealed that longitudinal and transverse polarized modes split in the frequency domain provided nonquasistatic effects are included. ${ }^{13}$ Waveguiding along the chain of gold nanoparticles with large bend radii on a metal surface was considered in Ref. 17. Studies of waveguiding on plasmonic metasurfaces was reviewed in Ref. 18. Despite the benefit of deep subwavelength field

\footnotetext{
a)andrey.miroshnichenko@anu.edu.au
}

localization provided by plasmonic nanostructures, light propagation in nanoparticle plasmonic waveguides is strongly limited by high dissipative losses. ${ }^{14,19,20}$

The CROW-type structures with the subwavelength guiding and low losses have been demonstrated with arrays of dielectric nanoparticles with high values of the refractive index. ${ }^{21-23}$ Such dielectric nanoparticles simultaneously support both magnetic dipole (MD) and electric dipole (ED) resonances, ${ }^{24-26}$ which give an additional control parameter over the light scattering. ${ }^{27-29}$ Recently, it was shown that waveguides composed of such nanoparticles support several different types of modes. ${ }^{23}$ In the case of spherical particles, the transversely polarized modes (with both MD and ED oriented perpendicular to the chain) and longitudinally polarized modes (when the dipoles are oriented along the chain) correspond to the same resonance frequencies, and they form separate propagation bands in different spectral regions, due to the different dipole-dipole interaction. For non-spherical shapes, corresponding dipole resonances depend on the orientation and aspect ratio of the particle. It allows to tune spectral range of propagation bands by changing the nanoparticle parameters. ${ }^{30,31}$

In this letter, we study the transmission of the CROWtype waveguides composed of one-dimensional (1D) arrays of high-index dielectric nanodisks of various configurations, as shown in Fig. 1. Such waveguides can be highly tuned due to presence of several independent parameters: $h$ and $d$ (height and diameter of the nanodisk, respectively) and $a$ (period of the chain). We consider dielectric disks with the fixed aspect ratio $2 h / d=1$. For such ratio, the frequencies of the MD resonance are well separated for different incident angles. Furthermore, we choose the appropriate period of the chain, so the longitudinal and transverse magnetic propagation bands spectrally overlap, which can not be achieved with spherical particles. As it will be demonstrated below, this is a key aspect allowing us efficiently transmit light through sharp $90^{\circ}$ bends. We support our theoretical conclusions by presenting experimental results for the microwave frequencies for an efficient guiding through $90^{\circ}$ bend in a microwave dielectric waveguide.

Waveguiding properties of CROW waveguides primarily depend on the modal structure of a single particle. 


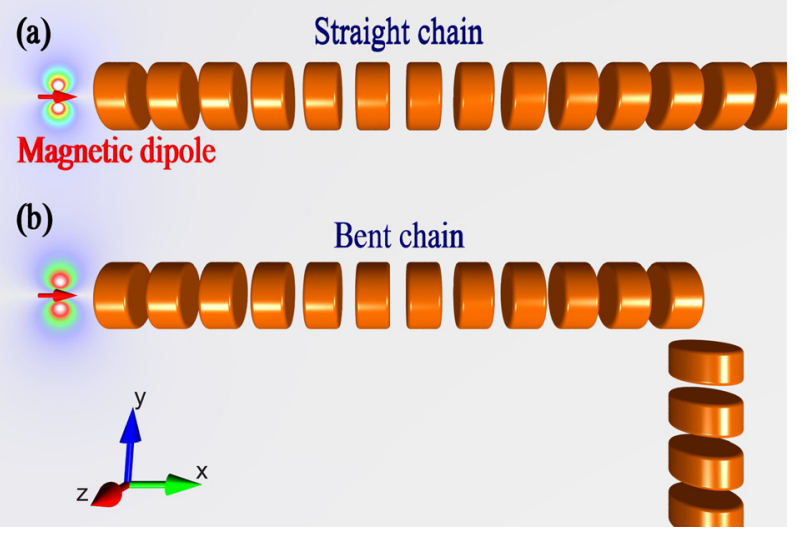

FIG. 1. Geometry of two types of all-dielectric waveguiding structures: (a) straight waveguide and (b) waveguide with a sharp bend. A magnetic dipole is employed as a source to excite the guided modes.

Therefore, we simulate the multipole field expansion for a single dielectric nanodisk with the fixed ratio $2 h / d=1$ and Si permittivity $\epsilon_{r}=16$ with the help of CST Microwave Studio, assuming that the disk is excited by a plane wave. The excited multipole moments of a nanodisk strongly depend on the incident wave direction and polarization. The scattering spectra for side and top incidence are presented in Figs. 2(a) and 2(b), respectively. In the case of the side incidence, the wave vector is oriented along the nanodisk axis,
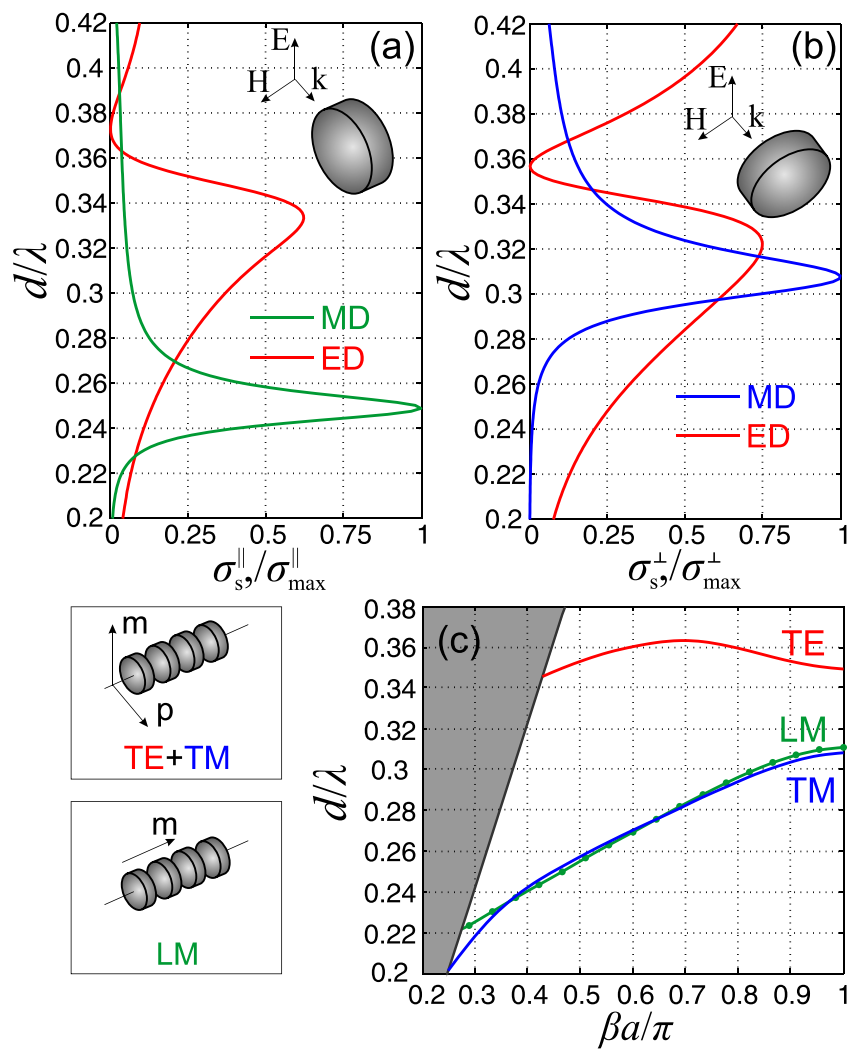

FIG. 2. (a) and (b) Multipole decomposition of the scattering spectra of a dielectric disk irradiated by a plane wave. Orientations of external polarization are shown in the insets. The partial scattering scattering cross-sections for two orientations $\sigma_{s}^{\perp, \|}$ are normalized to the corresponding maximal value $\sigma_{\max , \mathrm{MD}}^{\perp, \|}$ of the magnetic dipole contribution. (c) Dispersion diagram for an infinite chain of side-located nanodisks. Here, $d / \lambda$ is the normalized frequency; $d$ is the diameter of a nanodisk, and $\lambda$ is the wavelength; $\beta a / \pi$ is the normalized Bloch wavenumber, $a$ is the period of the chain. whereas for the top incidence, the wave vector is perpendicular to the nanodisk axis (see the insets). For the nanodisk under consideration, the fundamental resonance is $\mathrm{MD}$, and the second resonance is the ED. For the side incidence [see Fig. 2(a)], electromagnetic response of the nanodisk is similar to that of a sphere ${ }^{25}$ with diameter $d$ : the MD resonance is observed when wavelength inside the nanodisk matches its diameter $d \sqrt{\epsilon_{r}} / \lambda \approx 1$. Here, $\lambda$ is the wavelength in free space. For the top incidence, the MD and ED resonances approximately overlap when $d / \lambda \approx 0.31$ [see Fig. 2(b)], which is in full agreement with previous numerical calculations. ${ }^{30}$ The higher order multipole moments become significant only for higher frequencies $d / \lambda \gtrsim 0.45$, and therefore, we operate in the spectral range $d / \lambda \approx 0.2 \div 0.4$. Under those conditions, we can consider these dielectric nanodisks as hybrid magnetic and electric dipoles.

Figure 2(c) summarizes the waveguide modes of an infinite straight chain of nanodisks simulated by the eigenmode solver in CST Microwave Studio. Periodic boundary conditions have been applied in the $x$ direction, the electric boundary conditions have been used in both $y$ and $z$ directions. From those results, we identify (i) longitudinal magnetic (LM) mode that corresponds to the MD resonance in Fig. 2(a), (ii) transverse magnetic (TM) mode that corresponds to MD resonance in Fig. 2(b), and (iii) transverse electric (TE) mode that corresponds to the ED resonance in Fig. 2(b). Other modes fall out of the considered spectral region, and they are not shown in Fig. 2(c). By choosing the appropriate period of the chain $(a=1.25 h)$, we shift the LM and TM modes in such a way that they completely overlap, which provides an opportunity for efficient light transmittance in a chain with sharp bends.

Since the fabrication and measurement of optical nanodisk waveguides is rather complicated, here, we demonstrate the proof-of-concept experiments in the microwave frequency range, as has been done earlier for other dielectric structures. ${ }^{32,33}$ To this end, we scale up all the dimensions and perform, first, numerical simulations and, then, experimental studies of the wave transmission efficiency in the straight and sharp $90^{\circ}$ bent waveguides. In the microwave frequency range, $\mathrm{Si}$ nanoparticles are mimicked by $\mathrm{MgO}-$ $\mathrm{TiO}_{2}$ ceramic disks characterized with permittivity $\epsilon_{r}=16$ and dielectric loss factor of $0.1 \times 10^{-3}$, which can be measured at $10 \mathrm{GHz}^{32} \mathrm{We}$ consider both straight and bent chains composed of 30 ceramic disks with diameter $d=8 \mathrm{~mm}$ and height $h=4 \mathrm{~mm}$, placed on the distance $a=5 \mathrm{~mm}$ from each other. We note that if one considers a chain of silicon nanodisks in optical frequency range, the corresponding parameters should be close to the following: $d=160 \mathrm{~nm}, h=80 \mathrm{~nm}$, and $a=100 \mathrm{~nm}$.

First, we numerically simulate the transmission efficiency of straight waveguides composed of ceramic disks by employing CST Microwave Studio. We use two magnetic dipoles designed in CST Microwave Studio as a $2 \mathrm{~mm}$ coper loop with S-parameter port in $0.1 \mathrm{~mm}$ gap. The transmitting dipole is placed at $1 \mathrm{~mm}$ distance in front of the first disk, and the receiving dipole is placed $1 \mathrm{~mm}$ behind the last dielectric disk in such a way that the magnetic moments are oriented along the chain direction. The transmission efficiency of a straight waveguide simulated in the frequency 
range of $7-14 \mathrm{GHz}$ is shown in Fig. 3(a). We define the transmission efficiency as $\left|S_{21}\right|^{2} /\left|S_{21}\right|_{\max }^{2}$, where $\left|S_{21}\right|^{2}$ is the power transmitted from the first to second port normalized to the maximum value $\left|S_{21}\right|_{\max }^{2}$. According to the numerical results for a finite chain, the first pass-band is observed at $8-11.6 \mathrm{GHz}$ (normalized frequencies $0.21-0.3 d / \lambda$ ), which is in the excellent agreement with our theoretical prediction and the dispersion dependence [see Fig. 2(c)]. This propagation band can be explained by the presence of Fabry-Perot resonances of both LM and TM modes in a finite chain. The second pass-band corresponds to the TE mode of Fig. 2(c), and it appears in the frequency range $12.6-13.6 \mathrm{GHz}$ (normalized frequencies $0.33-0.36 d / \lambda$ ).

As a next step, we perform experimental study of the transmission efficiency of the straight waveguide of 30 ceramic disks. The disks are placed in a holder made of a styrofoam material with permittivity close to 1 . The magnetic dipoles are realized as coper loops with the diameter of $2 \mathrm{~mm}$ being connected to Agilent E8362C Vector Network Analyzer via coaxial cables. Transmitting and receiving loops are placed at $1 \mathrm{~mm}$ distance in front and behind of the first and last disks, respectively. The experimentally measured transmission efficiency is shown in Fig. 3(b), and it matches quite well with the numerical results. The experimentally measured pass-bands are observed at $8-11.4 \mathrm{GHz}$ and $12.6-13.6 \mathrm{GHz}$.

For a better understanding of the high-efficient transmission, we simulate numerically the magnetic field distribution in the straight waveguide at two characteristic frequencies, as shown in Figs. 3(c) and 3(d). Magnitude of the longitudinal $\left(H_{x}\right)$ and transverse $\left(H_{y}\right)$ components of the magnetic field at the lower edge of the first pass-band $(8 \mathrm{GHz})$ are negligibly small, and therefore, no transmission is observed. In the pass-band where both LM and TM modes simultaneously co-exist, the high-efficient transmission is observed. As an example, both $H_{x}$ and $H_{y}$ components of the magnetic field at $10 \mathrm{GHz}$ are strongly enhanced. The frequency range $11.6-12.6 \mathrm{GHz}$ corresponds to the stop-band, and therefore, no transmission is observed. In the second pass-band where only TE modes propagate, the magnitude of the longitudinal component of the magnetic field is close to zero, and for the demonstration, we show the fields at $12 \mathrm{GHz}$.

Finally, we study both numerically and experimentally the transmission efficiency of the waveguide with a $90^{\circ}$ bend. We consider again an all-dielectric waveguide composed of 30 ceramic disks with a bend in the middle. To get realistic efficiencies of the bent waveguides, we keep all the geometrical parameters as in the case of the straight waveguide. The numerical analysis is performed in the same way, assuming that the bent waveguide consists of two branches (horizontal and vertical) with equal number of disks in each of them. From the CST simulation, we obtain the $\left|S_{21}\right|^{2}$ parameter, and to estimate the transmission efficiency in this case, we normalize the obtained $\left|S_{21}\right|^{2}$ values to the values of $\left|S_{21}\right|_{\max }^{2}$ calculated for the straight waveguide, see Fig. 4(a). According to the numerical results and the measured data [see Fig. 4(b)], the pass-bands are observed at the frequencies $8-11.5 \mathrm{GHz}$ and $12.5-13.5 \mathrm{GHz}$. The maximum value of the transmission efficiency of the bent waveguide is up to 0.6 . The losses caused by the bend depend on the frequency, and they do not exceed $2.2 \mathrm{~dB}$. The efficient transmission through the $90^{\circ}$ bend is achieved due to an overlap of the LM and TM modes. As we can observe from Figs. 4(c) and 4(d), the LM mode in the horizontal branch transforms into the TM mode propagating in the vertical branch and vice versa.

We have analyzed theoretically and demonstrated experimentally the waveguiding properties of chains of high-index dielectric disks which support both electric and magnetic Mie-type dipole resonances. Our results indicate that the electromagnetic energy can be guided very efficiently in such all-dielectric structures even in the presence of sharp bends. We have proposed an alternative approach for the efficient transmission of subwavelength waveguides employing the mode polarization conversion. We have confirmed experimentally the guidance through a $90^{\circ}$ bend in the microwave waveguide and studied the structure of the guided fields. We believe that our approach can be generalized for designing optical waveguide structures based on alldielectric nanoparticles.
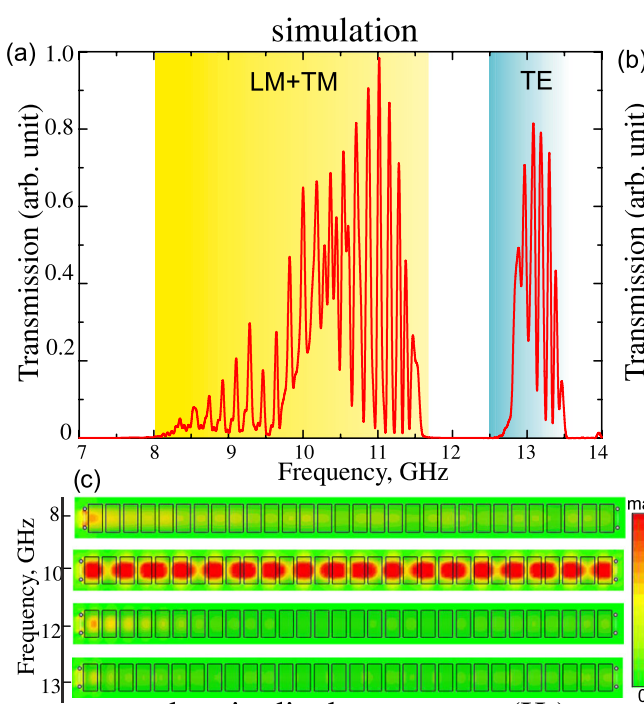

longitudinal component $\left(\mathrm{H}_{\mathrm{x}}\right)$
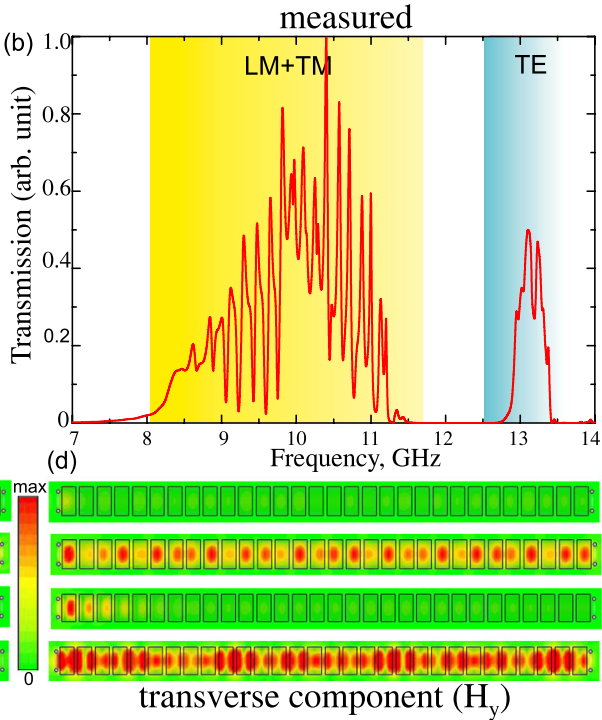

FIG. 3. (a) Numerically simulated and (b) experimentally measured transmittance of a straight array composed of 30 ceramic disks, as shown in Fig. 1. (c) and (d) Distribution of the $x$ - and $y$-components of the magnetic field for different frequencies. 


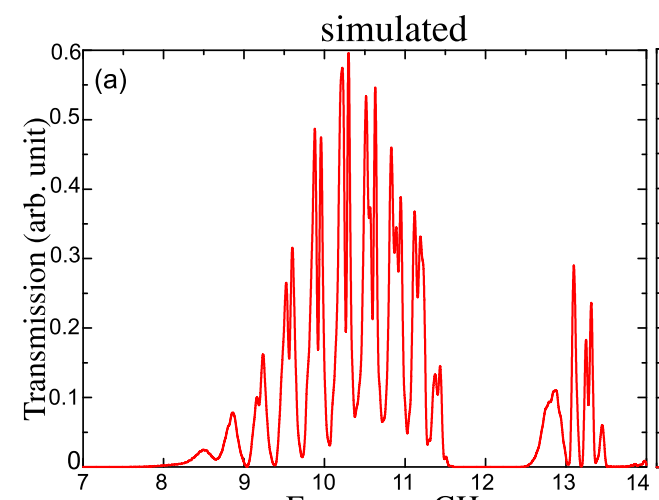

(c)

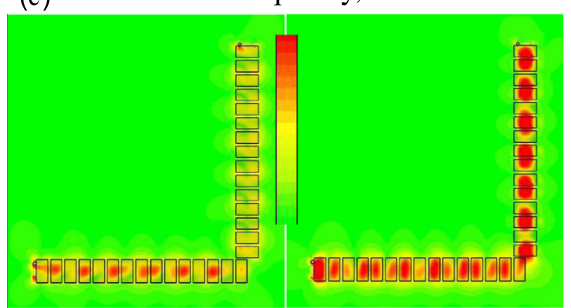

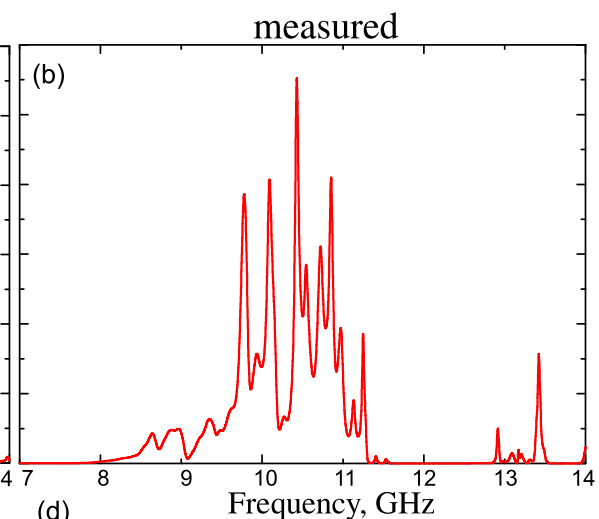

(d)

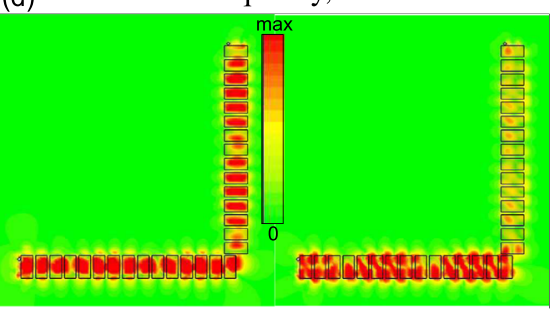

FIG. 4. (a) Numerically simulated and (b) experimentally measured transmission of a chain composed of 30 ceramic disks with a $90^{\circ}$ bend as a function of frequency. (c) and (d) Numerically simulated $x$ - and $y$-components of the magnetic field for the frequencies 9.2 $\mathrm{GHz}$ and $11.2 \mathrm{GHz}$, respectively.
This work was supported by the Ministry of Education and Science of the Russian Federation (Project 11.G34.31.0020, GOSZADANIE 2014/190, Zadanie No. 3.561.2014/K, 14.584.21.0009 10), by Russian Foundation for Basic Research, the Dynasty Foundation (Russia), the Australian Research Council via Future Fellowship Program (No. FT110100037), and the Australian National University. Study of the field distributions in nanostructured waveguides has been supported by the Russian Science Foundation Grant No. 14-12-01227.

${ }^{1}$ N. Daldosso and L. Pavesi, Laser Photonics Rev. 3, 508 (2009).

${ }^{2}$ C. Kopp, S. Bernabe, B. Bakir, J.-M. Fedeli, R. Orobtchouk, F. Schrank, H. Porte, L. Zimmermann, and T. Tekin, IEEE J. Sel. Top. Quantum Electron. 17, 498 (2011).

${ }^{3}$ Y. Chen, H. Li, and M. Li, Sci. Rep. 2, 622 (2012).

${ }^{4}$ L. Tong, R. Gattass, J. Ashcom, A. He, J. Lou, M. Chen, I. Maxwell, and E. Mazur, Nature 426, 816 (2003).

${ }^{5}$ M. Law, D. Sirbuly, J. Johnson, J. Goldberger, R. Saykally, and P. Yang, Science 305, 1269 (2004).

${ }^{6}$ K. Yamada, Silicon Photonics II, edited by D. Lockwood and L. Pavesi (Springer-Verlag Berlin Heidelberg, 2011).

${ }^{7}$ R. Espinola, R. Ahmad, F. Pizzuto, M. Steel, and R. Osgood, Opt. Express 8, 517 (2001).

${ }^{8}$ A. Mekis, J. C. Chen, I. Kurland, S. Fan, P. R. Villeneuve, and J. D. Joannopoulos, Phys. Rev. Lett. 77, 3787 (1996).

${ }^{9}$ M. Loncar, T. Doll, J. Vuckovic, and A. Sherer, J. Lightwave Technol. 18, 1402 (2000).

${ }^{10}$ A. Miroshnichenko and Y. Kivshar, Opt. Express 13, 3969 (2005).

${ }^{11}$ A. Yariv, Y. Xu, R. Lee, and A. Scherer, Opt. Lett. 24, 711 (1999).

${ }^{12}$ M. Quinten, A. Leitner, J. R. Krenn, and F. R. Aussenegg, Opt. Lett. 23, 1331 (1998).

${ }^{13}$ W. H. Weber and G. W. Ford, Phys. Rev. B 70, 125429 (2004).

${ }^{14}$ A. Alù and N. Engheta, Phys. Rev. B 74, 205436 (2006).
${ }^{15}$ M. L. Brongersma, J. W. Hartman, and H. A. Atwater, Phys. Rev. B 62, R16 356 (2000).

${ }^{16}$ S. A. Maier, M. L. Brongersma, P. G. Kik, S. Meltzer, A. A. G. Requicha, and H. A. Atwater, Adv. Mater. 13, 1501 (2001).

${ }^{17}$ A. B. Evlyukhin and S. I. Bozhevolnyi, Laser Phys. Lett. 3, 396 (2006).

${ }^{18}$ I. Radko, V. Volkov, J. Beermann, A. Evlyukhin, T. Søndergaard, A. Boltasseva, and S. Bozhevolnyi, Laser Photonics Rev. 3, 575 (2009).

${ }^{19}$ S. A. Maier, P. G. Kik, and H. A. Atwater, Appl. Phys. Lett. 81, 1714 (2002).

${ }^{20}$ D. Solis, B. Willingham, S. Nauert, L. Slaughter, J. Olson, P. Swanglap, A. Paul, W.-S. Chang, and S. Link, Nano Lett. 12, 1349 (2012).

${ }^{21}$ J. Du, S. Liu, Z. Lin, J. Zi, and S. T. Chui, Phys. Rev. A 79, 051801 (2009).

${ }^{22}$ J. Du, S. Liu, Z. Lin, J. Zi, and S. T. Chui, Phys. Rev. A 83, 035803 (2011).

${ }^{23}$ R. S. Savelev, A. P. Slobozhanyuk, A. E. Miroshnichenko, Y. S. Kivshar, and P. A. Belov, Phys. Rev. B 89, 035435 (2014).

${ }^{24}$ A. B. Evlyukhin, C. Reinhardt, A. Seidel, B. S. Lukyanchuk, and B. N. Chichkov, Phys. Rev. B 82, 045404 (2010).

${ }^{25}$ A. I. Kuznetsov, A. E. Miroshnichenko, Y. H. Fu, J. Zhang, and B. Lukyanchuk, Sci. Rep. 2, 492 (2012).

${ }^{26}$ A. B. Evlyukhin, S. M. Novikov, U. Zywietz, R. L. Eriksen, C. Reinhardt, S. I. Bozhevolnyi, and B. N. Chichkov, Nano Lett. 12, 3749 (2012).

${ }^{27}$ A. E. Krasnok, A. E. Miroshnichenko, P. A. Belov, and Y. S. Kivshar, Opt. Express 20, 20599 (2012).

${ }^{28}$ B. Rolly, B. Stout, and N. Bonod, Opt. Express 20, 20376 (2012).

${ }^{29}$ Y. H. Fu, A. I. Kuznetsov, A. E. Miroshnichenko, Y. F. Yu, and B. Lukyanchuk, Nat. Commun. 4, 1527 (2013).

${ }^{30}$ A. Evlyukhin, C. Reinhardt, and B. Chichkov, Phys. Rev. B 84, 235429 (2011).

${ }^{31}$ I. Staude, A. E. Miroshnichenko, M. Decker, N. T. Fofang, S. Liu, E. Gonzales, J. Dominguez, T. S. Luk, D. N. Neshev, I. Brener, and Y. Kivshar, ACS Nano 7, 7824 (2013).

${ }^{32}$ D. S. Filonov, A. E. Krasnok, A. P. Slobozhanyuk, P. V. Kapitanova, E. A. Nenasheva, Y. S. Kivshar, and P. A. Belov, Appl. Phys. Lett. 100, 201113 (2012).

${ }^{33}$ G. Boudarham, R. Abdeddaim, and N. Bonod, Appl. Phys. Lett. 104, 021117 (2014). 